\title{
A completely entangled subspace of maximal dimension
}

\author{
B. V. Rajarama Bhat \\ Indian Statistical Institute, \\ R. V. College Post, Bangalore 560059, India. \\ E-mail: bhat@isibang.ac.in
}

September 1, 2004

\begin{abstract}
Consider a tensor product $\mathcal{H}=\mathcal{H}_{1} \otimes \mathcal{H}_{2} \otimes \cdots \otimes \mathcal{H}_{k}$ of finite dimensional Hilbert spaces with dimension of $\mathcal{H}_{i}=d_{i}, 1 \leq i \leq k$. Then the maximum dimension possible for a subspace of $\mathcal{H}$ with no non-zero product vector is known to be $d_{1} d_{2} \ldots d_{k}-\left(d_{1}+d_{2}+\cdots+d_{k}\right)+k-1$. We obtain an explicit example of a subspace of this kind. We determine the set of product vectors in its orthogonal complement and show that it has the minimum dimension possible for an unextendible product basis of not necessarily orthogonal product vectors.
\end{abstract}

KEY WORDS: completely entangled subspaces, unextendible product bases, separable states, entangled states.

Mathematics Subject Classification: 81P68, 15 A03.

Let $\mathcal{H}_{i}, l \leq i \leq k$ be finite dimensional complex Hilbert spaces with $\operatorname{dim}\left(\mathcal{H}_{i}\right)$ $=d_{i}, \quad 1 \leq i \leq k$. To avoid trivialities we assume that $d_{i} \geq 2$ for every $i$. A state $\rho$ on the tensor product space $\mathcal{H}=\mathcal{H}_{1} \otimes \ldots \otimes \mathcal{H}_{k}$ is said to be separable if it is a convex combination of product states. Otherwise it is said to be 
entangled. Entangled states play an important role in quantum computation and quantum coding theories. However in general it is not easy to decide whether a state is entangled or not. It is a result of Horodecki and Horodecki [1] that if a state is separable then its support is spanned by product vectors (vectors of the form $y_{1} \otimes \ldots \otimes y_{k}$ ) in the support. In this context K.R. Parthasarathy [4] defines a subspace $\mathcal{S} \subset \mathcal{H}$ as completely entangled if it does not contain a single non-zero product vector. This in particular means that any state $\rho$ with its support in $\mathcal{S}$ is automatically entangled. So the construction of entangled states becomes easy if we have explicit completely entangled subspaces at our disposal.

The notion of completely entangled subspaces is very closely connected with notions of unextendible product bases and uncompletable product bases introduced earlier by D. P. Vincenzo et al. [2]. We say that a linearly independent subset $B$ of prodcut vectors $\mathcal{H}$ forms an unextendible product basis (UPB) if $B^{\perp}$ is completely entangled. If in addition the vectors in $B$ are mutually orthogonal we call it an orthogonal UPB. It is to be noted that in [2] only orthogonal bases are considered. This difference is crucial (In this respect see also Pittenger [5] ). Through some interesting combinatorics and number theory it was shown by Alon and Lovász [3] that the minimum dimension possible for an orthogonal UPB is $N+1$, where $N=\sum_{i}\left(d_{i}-1\right)$, unless either (i) $k=2$ and $2 \in\left\{d_{1}, d_{2}\right\}$; or (ii) $N+1$ is odd and at least one $d_{i}$ is even. In each of these two special cases, the minimum dimension possible for an orthogonal UPB is strictly larger than $N+1$. In contrast to this here in Corollary 4 we show that the minimum dimension possible for an UPB (of not necessarily orthogonal vectors) is always $N+1$. We make use of the basic result from Parthasarathy [4] that the maximal dimension possible for a completely entangled subspaces is $d_{1} d_{2} \ldots d_{k}-(N+1)$.

Parthasarathy obtains an explicit example of a completely entangled subspace of maximal dimension and exhibits an orthonormal basis for it only in the very special case of $k=2$ and $d_{1}=d_{2}=n$. Even in this specail case the basis he obtains is quite complicated. In this short note we give a very simple construction of a completely entangled subspace of maximal dimension. Further we show that the orthogonal complement of this space is spanned by product vectors.

To simplify notation we fix an infinite dimensional Hilbert space $\mathcal{K}$ with ortho-normal basis $\left\{e_{0}, e_{1}, \ldots,\right\}$ and identify $\mathcal{H}_{i}$ with $\operatorname{span}\left\{e_{0}, e_{1} \ldots, e_{d_{i-1}}\right\}$, 
so that for each $i, \quad\left\{e_{0}, e_{1}, \ldots, e_{d_{i-1}}\right\}$ is an otho-normal basis for $\mathcal{H}_{i}$. Now take

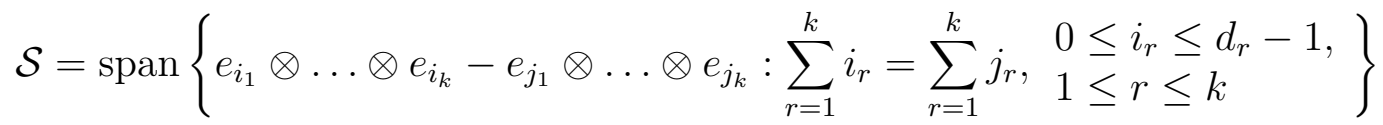

We claim that $\mathcal{S}$ is a completely entangled subspace of maximal dimension. In order to analyze the structure of $\mathcal{S}$ we first identify $\mathcal{S}^{\perp}$. Note that $\mathcal{H}$ decomposes as $\mathcal{H}=\bigoplus_{n=0}^{N} \mathcal{H}^{(n)}$, where

$$
\mathcal{H}^{(n)}=\operatorname{span}\left\{e_{i_{1}} \otimes \ldots \otimes e_{i_{k}}: \quad \sum_{r=1}^{k} i_{r}=n\right\} .
$$

Further observe that if $\mathcal{M}$ is a Hilbert space with orthonormal basis $\left\{x_{1}\right.$, $\left.\ldots, x_{p}\right\}$ and $\mathcal{N}=\operatorname{span}\left\{x_{i}-x_{j}: 1 \leq i, j \leq p\right\}$, then $N^{\perp}=\mathbb{C} u$, where $u=x_{1}+\ldots+x_{p}$. Now it is easy to see that each $\mathcal{H}^{(n)}$ decomposes as $\mathcal{H}^{(n)}=\mathcal{S}^{(n)} \oplus \mathcal{T}^{(n)}$, where

$$
\begin{gathered}
\mathcal{T}^{(n)}=\mathbb{C} u_{n}, \quad u_{n}=\sum_{i_{1}+\ldots+i_{k}=n} e_{i_{1}} \otimes \ldots \otimes e_{i_{k}} \\
\mathcal{S}^{(n)}=\operatorname{span}\left\{e_{i_{1}} \otimes \ldots \otimes e_{i_{k}}-e_{j_{1}} \otimes \ldots e_{j_{k}}: \quad \sum_{r=1}^{k} i_{r}=\sum_{r=1}^{k} j_{r}=n\right\} . \\
\mathcal{S}^{(0)}=\mathcal{S}^{(N)}=\{0\} . \text { Moreover } \mathcal{S}=\bigotimes_{n=0}^{N} \mathcal{S}^{(n)}, \text { and } \mathcal{S}^{\perp}=\bigoplus_{n=0}^{N} \mathcal{T}^{(n)} .
\end{gathered}
$$

Theorem 1: $\mathcal{S}$ is a completely entangled subspace of $\mathcal{H}_{1} \otimes \ldots \otimes \mathcal{H}_{k}$, of maximal dimension.

Proof : As each $\mathcal{T}^{(n)}$ is one dimensional, we see that $\operatorname{dim}(\mathcal{S})=d_{1} d_{2} \ldots d_{k}-$ $\operatorname{dim}\left(\mathcal{S}^{\perp}\right)=d_{1} d_{2} \ldots d_{k}-(N+1)$. Now suppose $x_{1} \otimes \ldots \otimes x_{k}$ is a non-zero product vector in $\mathcal{S}$. Then for any $\lambda \in \mathbb{C}, \quad\left\langle x_{1} \otimes \ldots \otimes x_{k}, \quad \sum_{n=0}^{N} \lambda^{n} u_{n}\right\rangle=0$. However,

$\sum_{n=0}^{N} \lambda^{n} u_{n}=\sum_{n=0}^{N} \sum_{i_{1}+\ldots+i_{k}=n} \lambda^{i_{1}+\ldots+i_{k}} e_{i_{1}} \otimes \ldots \otimes e_{i_{k}}=y_{1}^{\lambda} \otimes \ldots \otimes y_{k}^{\lambda}$, where $y_{r}^{\lambda}=\sum_{j=0}^{d_{r}-1} \lambda^{j} e_{j}, \quad$ for $1 \leq r \leq k$. So on one hand we get $\prod_{r=1}^{k}\left\langle x_{r}, y_{r}^{\lambda}\right\rangle=0$ for all $\lambda \in \mathbb{C}$.

On the other hand by basic properties of van der Monde matrix $\left\{y_{r}^{\lambda}\right\}$ are linearly independent for any $d_{r}$, distinct $\lambda$ 's. So $\#\left\{\lambda:\left\langle x_{r}, y_{r}^{\lambda}\right\rangle=0\right\} \leq$ 
$d_{r}-1<\infty$ for every $r$. Hence $\#\left\{\lambda: \prod_{r=1}^{k}\left\langle x_{r}, y_{r}^{\lambda}\right\rangle=0\right\}$ must be finite. This is a contradiction. Therefore $\mathcal{S}$ has no non-zero product vector.

Usually for computational purposes one needs an explicit orthonormal basis for the entangled space under consideration. Obtaining simple orthonormal bases for our $\mathcal{S}$ is not difficult as it suffices to get orthonormal bases for each $\mathcal{S}^{(n)}$. As $\mathcal{S}^{(n)}$ is the space orthogonal to $u_{n}$ in $\mathcal{H}^{(n)}$, there are many ways we can obtain a basis for it. All we need to do is obtain a basis for $\mathcal{H}^{(n)}$ where $u_{n}$ is one of the vectors. For instance we get one such basis by considering non-trivial characters of an abelian group of order $a_{n}\left(d_{1}, \ldots, d_{k}\right)$ (the trivial character gets identified with $\left.u_{n}\right)$, where $a_{n}\left(d_{1}, \ldots, d_{k}\right)$ is the dimension of $\mathcal{H}^{(n)}$.

From the definition of $\mathcal{H}^{(n)}$ it is clear that

$$
a_{n}\left(d_{1}, \ldots, d_{k}\right)=\#\left\{\left(i_{1}, \ldots, i_{k}\right): i_{1}+\ldots+i_{k}=n, \quad 0 \leq i_{r} \leq d_{r}-1\right\} .
$$

In other words, $a_{n}\left(d_{1}, \ldots, d_{k}\right)$ is the coefficient of $x^{n}$ in the polynomial

$$
p(x)=\prod_{r=1}^{k}\left(1+x+x^{2}+\ldots+x^{d_{r}-1}\right) .
$$

In particular if $k=2$, and $d_{1} \leq d_{2}$, then

$$
a_{n}\left(d_{1}, d_{2}\right)= \begin{cases}n+1 & \text { if } \quad 0 \leq n \leq d_{1}-1 \\ d_{1} & \text { if } \quad d_{1}-1<n \leq d_{2}-1 \\ d_{1}+d_{2}-(n+1) & \text { if } \quad d_{2}-1<n \leq d_{1}+d_{2}-2\end{cases}
$$

And in the case of qubits i.e., if $d_{i} \equiv 2, \quad 1 \leq i \leq k$, then

$$
a_{n}\left(d_{1}, \ldots, d_{k}\right)=\left(\begin{array}{c}
k \\
n
\end{array}\right), \quad 0 \leq n \leq k .
$$

When $k=2$, by identifying $\mathcal{H}_{1} \otimes \mathcal{H}_{2}$ with $d_{1} \times d_{2}$ matrices in the usual way (identify $e_{i} \otimes e_{j}$ with matrix unit $E_{i j}$ ) and noting that in this identification non-zero product vectors correspond to rank one matrices we arrive at the following Example.

Example 1: Consider the vector space $M_{d_{1}, d_{2}}$ of $d_{1} \times d_{2}$ complex matrices. If a subspace of $M_{d_{1}, d_{2}}$ has no rank one element then it has dimension atmost 
$\left(d_{1}-1\right)\left(d_{2}-1\right)$. One such subspace of maximal dimension is given by:

$$
\left\{\left[a_{i j}\right]: \sum_{i+j=n} a_{i j}=0 \quad \forall n\right\} .
$$

Now we determine product vectors of $\mathcal{S}^{\perp}$.

Theorem 2: The set of product vectors in $\mathcal{S}^{\perp}$ is $\left\{c z^{\lambda}: c \in \mathbb{C}, \lambda \in \mathbb{C} \cup\{\infty\}\right\}$, where

$$
\begin{aligned}
z^{\lambda} & =\bigotimes_{r=1}^{k}\left(e_{0}+\lambda e_{1}+\ldots+\lambda^{d_{r}-1} e_{r}\right) \quad \lambda \in \mathbb{C} \\
z^{\infty} & =\bigotimes_{r=1}^{k} e_{d_{r}-1}
\end{aligned}
$$

Proof: Consider arbitrary vectors $y_{r}=\sum_{i=0}^{d_{r-1}} a_{i}^{r} e_{i}$, in $\mathcal{H}_{r}$ for $1 \leq r \leq k$. If $y_{1} \otimes y_{2} \otimes \ldots \otimes y_{k} \in \mathcal{S}^{\perp}$, we obtain

$$
a_{i_{1}}^{1} a_{i_{2}}^{2} \ldots a_{i_{k}}^{k}=a_{j_{1}}^{1} a_{j_{2}}^{2} \ldots a_{j_{k}}^{k}
$$

whenever $\sum i_{r}=\sum j_{r}$.

Case(i): $a_{0}^{1} a_{0}^{2} \ldots a_{0}^{k} \neq 0$. In this case we may take $a_{0}^{r}=1$, for $1 \leq r \leq k$. Take $\lambda=a_{1}^{1}$. Now (1.3) applied to $k$-tuples of the form $(1,0,0, \ldots, 0),(0,1,0$, $\ldots 0)$, i.e, those $k$-tuples with $\sum i_{r}=\sum j_{r}=1$, gives $a_{1}^{r}=\lambda, \forall r$. Then by considerting $k$-tuples with $\sum i_{r}=\sum j_{r}=2$ we get $a_{2}^{r}=\lambda^{2}, \forall r$. Continuing this way we finally obtain $y_{1} \otimes \ldots \otimes y_{k}=z^{\lambda}$.

Case (ii): $a_{0}^{1} a_{0}^{2} \ldots a_{0}^{k}=0$. Now for $1 \leq r \leq k$, let $j_{r}$ be the smallest $j$ such that $a_{j}^{r} \neq 0$. So $a_{j_{1}}^{1} a_{j_{2}}^{2} \ldots a_{j_{k}}^{k} \neq 0$. Now if for some $r \neq s, j_{r}>0$ and $j_{s}<d_{s}-1$. By (1.3) we get $a_{j_{1}}^{1} a_{j_{2}}^{2} \ldots a_{j_{r}-1}^{r} \ldots a_{j_{s}+1}^{s} \ldots a_{j_{k}}^{k}=a_{j_{1}}^{1} a_{j_{2}}^{2} \ldots a_{j_{k}}^{k} \neq 0$. But this is not possible as $a_{j_{r}-1}^{r}=0$. In other words if $j_{r}>0$, then $j_{s}=d_{s}-1$ for all $s \neq r$. Interchanging the role of $r$ and $s$ we see that $j_{r}=d_{r}-1$ for all $r$. This shows that $y_{1} \otimes \ldots \otimes y_{k}=c z^{\infty}$, for some $c \in \mathbb{C}$.

It may be noted that $\lim _{|\lambda| \rightarrow \infty} \frac{z^{\lambda}}{(\lambda)^{N}}=z^{\infty}$. This justifies the notation used in Theorem 2. 
Theorem 3: Any $N+1$ non-zero product vectors in $\mathcal{S}^{\perp}$ span whole of $\mathcal{S}^{\perp}$ and hence form an unextendible product basis.

Proof: Consider $B \subset \mathbb{C} \cup\{\infty\}$, with $\# B=N+1$. Let $x=\sum_{n=0}^{N} c_{n} u_{n}$, be an arbitrary vector in $\mathcal{S}^{\perp}$ such that $\left\langle x, z^{\lambda}\right\rangle=0 \quad \forall \lambda \in B$. We need to show that $x=0$.

Case (i): $B \subset \mathbb{C}$. Note that

$q(\lambda):=\left\langle x, z^{\lambda}\right\rangle=\left\langle\sum c_{n} u_{n}, z^{n}\right\rangle=\sum_{n=0}^{N} \bar{c}_{n} a_{n}\left(d_{1}, \ldots, d_{k}\right) \lambda^{n}$.

So $q$ is a polynomial in $\lambda$ of degree atmost $N$. Therefore if $q(\lambda)=0 \forall \lambda \in B$, as $\# B=N+1$, we get $q=0$. In otherwords $c_{n} \equiv 0$, or $x=0$.

Case (ii): $\infty \in B$. Here $\left\langle x, z^{\infty}\right\rangle=C_{N}=0$. So for $\lambda \in \mathbb{C}, \quad q(\lambda)=\left\langle x, z^{\lambda}\right\rangle$ is a polynomial of degree atmost $(N-1)$. Now we can argue as in Case(i).

It is to be noted that $\mathcal{S}^{\perp}$ may not contain any unextendible product basis consisting of orthogonal product vectors. This can be seen by taking simple examples such $k=2, d_{1}=2, d_{2}=3$, or by making use of beautiful results of Alon and Lovász[3], where the minimum dimension of an unextendible product basis consisting of orthogonal vectors is seen to be strictly larger than $\left(d_{1}+\ldots+d_{k}-k+1\right)$ in some special cases.

Corollary 4: The minimal dimension of unextendible product bases (of not necessarily orthogonal vectors) in $\mathcal{H}_{1} \otimes \ldots \mathcal{H}_{k}$ is $d_{1}+d_{2}+\ldots+d_{k}-k+1$.

Proof: If $B$ is an unextendible product basis, then $B^{\perp}$ is completely entangled. Hence by Parthasarathy [4], $\operatorname{dim}\left(B^{\perp}\right) \leq d_{1} \ldots d_{k}-(N+1)$, or $\operatorname{dim}(\operatorname{span} B) \geq N+1$. Further we have shown that that there exists an unextendible basis of dimension $(N+1)$.

Now it is a natural question as to what are the possible dimensions of unextendible bases. We do not have the answer in general. But here is the answer when $k=2$.

Theorem 5: Suppose $k=2$. Then for any $m$ with $d_{1}+d_{2}-1 \leq m \leq d_{1} d_{2}$, there exists an unextendible product basis of dimension $m$. 
Proof : Consider the decomposition of $\mathcal{H}$ as $\mathcal{H}=\bigoplus_{n=0}^{N} \mathcal{H}^{(n)}=\bigoplus_{n=0}^{N}\left(\mathcal{S}^{(n)} \oplus \mathcal{T}^{(n)}\right)$. We know that $\mathcal{S}$ is completely entangled, so any subspace of it is also completely entangled. $\mathcal{S}^{\perp}$ has a product basis, and also by the definition of $\mathcal{H}^{(n)}, \mathcal{S}^{(n)} \oplus \mathcal{T}^{(n)}$, also has a product basis for every $n$. Therefore $\mathcal{S}^{\perp} \cup \mathcal{S}^{(n)}=$ $\mathcal{S}^{\perp} \cup\left(\mathcal{S}^{(n)} \cup \mathcal{T}^{(n)}\right)$ also has a product basis. Similarly $\mathcal{S}^{\perp} \cup\left(\bigcup_{n \in M} \mathcal{S}^{(n)}\right)$ also has a product basis for any subset $M$ of $\left\{0,1, \ldots, d_{1}+d_{2}-1\right\}$.

As $\operatorname{dim}\left(\bigcup_{n \in M} \mathcal{S}^{(n)}\right)=\sum_{n \in M} \operatorname{dim}\left(\mathcal{S}^{(n)}\right)=\sum_{n \in M}\left(a_{n}\left(d_{1}, d_{2}\right)-1\right)$, and the formula for $a_{n}\left(d_{1}, d_{2}\right)$ is as in (1.1), we may choose a suitable set $M$ such that $\operatorname{dim}$ $\left(\bigcup_{n \in M} \mathcal{S}^{(n)}\right)=m-\left(d_{1}+d_{2}-1\right)$. Now it should be clear that any product basis of $\mathcal{S}^{\perp} \cup\left(\bigcup_{n \in M} \mathcal{S}^{(n)}\right)$ does the job.

Here is an example to show that in general even if a subspace $\mathcal{M}$ is completely entangled $\mathcal{M}^{\perp}$ may not be spanned by product vectors. In this case states with support equal to $\mathcal{M}^{\perp}$, as well as states with support equal to $\mathcal{M}$ are entangled!

Example 2: Take $k=2, d_{1}=d_{2}=4$, and identify $\mathcal{H}_{1} \otimes \mathcal{H}_{2}$ with $4 \times 4$ matrices as in Example 1. Now take

$$
\begin{aligned}
\mathcal{M}=\left\{\left[a_{i j}\right]_{0 \leq i, j \leq 3}: 0\right. & =a_{00}=a_{01}+a_{10}=a_{02}+a_{11}+a_{20}=a_{03}+a_{12} \\
& \left.=a_{21}+a_{30}=a_{13}+a_{22}+a_{31}=a_{23}+a_{32}=a_{33}\right\}
\end{aligned}
$$

Then

$$
\left.\begin{array}{c}
\mathcal{M}^{\perp}=\left\{\left[a_{i j}\right]_{0 \leq i, j \leq 3}: a_{01}=a_{10}, a_{02}=a_{11}=a_{22}, a_{03}=a_{12},\right. \\
\\
a_{21}=a_{30}, a_{13}=a_{22}=a_{31}, a_{23}=a_{32}
\end{array}\right\}
$$

It is easily seen that the set of rank one elements in $\mathcal{M}^{\perp}$ is given by $\mathcal{R}=\left\{c\left[\lambda^{i+j}\right]_{0 \leq i, j \leq 3}: c \in \mathbb{C}, \lambda \in \mathbb{C}\right\} \cup\left\{c\left[a_{i j}\right]: a_{33}=1, a_{i j}=0\right.$ otherwise $\}$ and $\mathcal{R}$ does not $\operatorname{span} \mathcal{M}^{\perp}$.

Note that $\mathcal{R}^{\perp}=\left\{\left[a_{i j}\right]: \sum_{i+j=n} a_{i j}=0 \quad \forall n\right\}=\mathcal{S}$, which is completely entangled. So $\mathcal{R}$ still forms an unextendible product basis in $\mathcal{H}_{1} \otimes \mathcal{H}_{2}$.

Acknowledgement: This work was supported by the Department of Science and Technology (India), under Swarnajayanthi Fellowship Project. 


\section{References}

[1] M. Horodecki and R. Horodecki, Separability of mixed states : necessary and sufficient conditions, Phys. Lett. A 223 (1-2), 1-8, 1996.

[2] D. P. Di Vincenzo, T. Mor, P. W. Shor, J. A. Smolin, B. M. Terhal, Unextendible product Bases, Uncompletable Product Bases and Bound Entanglement, Comm. Math. Phys. 238, 379-410(2003). quantph/9908070.

[3] N. Alon, L. Lovász, Unextendible product bases, preprint,

http://www . math.tau.ac.il/ nogaa/PDFS/shor5.pdf .

[4] K. R. Parthasarathy, On the maximal dimension of a completely entangled subspace for finite level quantum systems, quant-ph/0405077.

[5] Arthur O. Pittenger, Unextendible product bases and the construction of inseparable states, Linear Algebra and its Applications 359, 235248(2003). quant-ph/0208028. 